\title{
The Outbreak of Avian Influenza A (H7N9) in China: Current Status and Future Prospects
}

\author{
Lu Lu${ }^{1}$, Biao $\mathrm{He}^{2}$, Shibo Jiang ${ }^{3}$
}

$\mathrm{I}_{\mathrm{a}}^{\mathrm{n}}$ March 2013, an influenza outbreak caused by novel influenza A (H7N9) virus occurred in China. ${ }^{[1-3]}$ As of May 29, 2013, 132 confirmed cases, including 37 deaths (a case fatality rate of $28 \%$ ), were reported to the World Health Organization. Since then, no new cases of A (H7N9) have been reported. ${ }^{[4]}$ Can it be presumed that this A (H7N9) outbreak is over and, if so, will it re-emerge?

At this stage, no one can claim that the outbreak is completely over; however, in the absence of evidence to the contrary, it appears to have reached its peak. Nonetheless, public health authorities and researchers in China continue to maintain enhanced surveillance, and epidemiological investigation is ongoing, in order to more clearly define the origin of the virus, monitor its spread, and develop prevention and control measures.

Genomic signature analysis has confirmed the avian origin of A (H7N9), revealing 43 avian signatures to only 4 human signatures. ${ }^{[3]}$ Phylogenetic analysis further indicates that its HA gene might have originated from $\mathrm{H} 7 \mathrm{~N} 3$ in ducks in Zhejiang Province, whereas its NA gene might have originated from migratory birds along the East Asian flyway. Its six internal genes may originate from H9N2 avian influenza viruses (AIVs) isolated from chickens and birds, such as bramblings in Beijing. Therefore, ducks and chickens in the Yangtze River Delta (YRD) region could have served as the intermediate hosts for this virulent A (H7N9) virus. ${ }^{[5]}$ Based on this evidence, researchers are asking how migratory birds passed the virus to poultry. One theory proposed by Li et al., ${ }^{[2]}$ suggests that wild ducks first transmitted the virus to domesticated ducks, as both have similar patterns of behavior and habitats in the YRD, further passing it on to chickens. Chongming Island near Shanghai is the home of many wild birds. This may explain why Shanghai was the most affected area during the initial outbreak of A (H7N9). ${ }^{[6]}$

Unlike the highly pathogenic AIV, A (H5N1), which has killed millions of birds, A (H7N9) still has low patho- genicity to birds and poultry, which thus act as hidden reservoirs for this zoonotic A (H7N9) virus. The live poultry markets in YZD have been confirmed to be the major risk factor for human infection of A (H7N9) virus since the virus was identified from the live poultry and the environment in these markets, ${ }^{[7]}$ and closing the live poultry markets resulted in significant reduction of morbidity rate of A (H7N9).

Due to the lack of a vaccine against A (H7N9) virus, the prophylactic measures that should be taken may include: (1) staying away from live poultry markets and the surrounding environment; (2) avoiding touching live birds and their excretions or secretions; (3) performing hand hygiene; (4) maintaining respiratory hygiene; and (5) taking food safety measures.

A (H5N1) and other AIVs have shown a seasonal pattern whereby human cases in summer months are much lesser than those in winter months. We expect that A (H7N9) will follow the same seasonal pattern. However, since migratory birds were first implicated in H7N9 transmission, we cannot exclude the possibility that the virus may spread into other regions or countries with colder weather, given the widespread migratory patterns, thus causing a new outbreak there.

We now ask whether we should expect its re-emergence during the winter months and, if so, what precautionary steps should be taken. A global surveillance system should be established to monitor the spread of AIVs, particularly A (H7N9) and A (H5N1). Close collaboration between the epidemiologists responsible for monitoring AIVss in humans and those monitoring AIVs in migratory birds, poultry, and the environment is urgently needed. It is also important for researchers to identify the viral mutations or reassortments that may alter risks for animals or humans, in order to gain insight into critical viral characteristics, including antiviral resistance, transmissibility, and pathogenicity, followed by developing strategies to control the future spread of A (H7N9).

From the ${ }^{1}$ Key Laboratory of Medical Molecular Virology of Ministries of Education and Health, Shanghai Medical College and Institute of Medical Microbiology, Fudan University, Shanghai, China; ${ }^{2}$ College of Life Sciences, Hunan Normal University, Changsha, Hunan, China; ${ }^{3}$ Lindsley F. Kimball Research Institute, New York Blood Center, New York, NY, USA

Received: May 20, 2013; Accepted: May 27, 2013

Correspondence to: Dr. Shibo Jiang, Key Lab of Medical Molecular Virology of Ministries of Education and Health Shanghai Medical College, Fudan University. 130 Dong An Road, Building \#13, Rm 611 Xuhui District, Shanghai 200032, China. Tel: 86-21-54237673; Fax: 86-21-54237465; E-mail: shibojiang@ fudan.edu.cn

DOI: $10.4103 / 2319-4170.113224$ 


\section{REFERENCES}

1. Gao R, Cao B, Hu Y, Feng Z, Wang D, Hu W, et al. Human infection with a novel avian-origin influenza A (H7N9) virus. N Engl J Med 2013;368:1888-97.

2. Liu D, Shi W, Shi Y, Wang D, Xiao H, Li W, et al. Origin and diversity of novel avian influenza A H7N9 viruses causing human infection: Phylogenetic, structural, and coalescent analyses. Lancet 2013;381;1926-32.

3. Liu Q, Lu L, Sun Z, Chen GW, Wen Y, Jiang S. Genomic signature and protein sequence analysis of a novel influenza A(H7N9) virus that causes an outbreak in humans in China. Microbes Infect 2013 [Epub ahead of print].
4. WHO. Human infection with avian influenza A (H7N9) virus in China-update. Available from: http://www.who.int/csr/ don/2013_05_29/en/index.html. [Last accessed on 2013 May 29].

5. Kageyama T, Fujisaki S, Takashita E, Xu H, Yamada S, Uchida Y, et al. Genetic analysis of novel avian A (H7N9) influenza viruses isolated from patients in China, February to April 2013. Euro Surveill 2013;18. pii: 20453.

6. Butler D. Receptor for new coronavirus found. Nature 2013;495:149-50.

7. Chen Y, Liang W, Yang S, Wu N, Gao H, Sheng J, et al. Human infections with the emerging avian influenza A H7N9 virus from wet market poultry: Clinical analysis and characterisation of viral genome. Lancet 2013;381:1916-25. 